\title{
Viscous fingering as a paradigm of interfacial pattern formation: Recent results and new challenges
}

\author{
Jaume Casademunt ${ }^{\mathrm{a})}$ \\ Departament d'Estructura i Constituents de la Matèria, Universitat de Barcelona, Avinguda Diagonal, 647, \\ 08028 Barcelona, Spain
}

(Received 27 February 2004; accepted 1 July 2004; published online 16 September 2004)

\begin{abstract}
We review recent results on dynamical aspects of viscous fingering. The Saffman-Taylor instability is studied beyond linear stability analysis by means of a weakly nonlinear analysis and the exact determination of the subcritical branch. A series of contributions pursuing the idea of a dynamical solvability scenario associated to surface tension in analogy with the traditional selection theory is put in perspective and discussed in the light of the asymptotic theory of Tanveer and co-workers. The inherently dynamical singular effects of surface tension are clarified. The dynamical role of viscosity contrast is explored numerically. We find that the basin of attraction of the Saffman-Taylor finger depends on viscosity contrast, and that the sensitivity to this parameter is maximal in the usual limit of high viscosity contrast. The competing attractors are identified as closed bubble solutions. We briefly report on recent results and work in progress concerning rotating Hele-Shaw flows, topological singularities and wetting effects, and also discuss future directions in the context of viscous fingering. (C) 2004 American Institute of Physics. [DOI: 10.1063/1.1784931]
\end{abstract}

The study of viscous fingering in a Hele-Shaw cell is a longstanding problem which has become an archetype of interfacial pattern formation, but continues to bring up new surprises which challenge our understanding of nonlocal, nonlinear pattern dynamics of interfaces. The problem refers to the dynamics of the interface between two immiscible viscous fluids confined in a quasi-twodimensional geometry, the Hele-Shaw cell, leading to pattern formation through a morphological instability. In this article we briefly review some recent developments on the dynamics of fingering patterns. We discuss the effects of surface tension as a singular perturbation showing that the problem with and without surface tension are essentially different. Within a dynamical systems approach, we describe how the introduction of surface tension dramatically modifies the global (topological) structure of the phase space flow of the system. We also address, in more detail, the effect of varying the parameter viscosity contrast. We show that the dynamics of fingered structures is highly sensitive to this parameter, and that the long time asymptotics is dominated by the competition between the usual Saffman-Taylor single-finger stationary solution and other attractors defined by closed bubbles. In this context and also taking into account recent results on rotating Hele-Shaw flows, we discuss future perspectives in the field concerning the existence of topological singularities in the form of interface pinchoff, wetting effects and applications to other problems such interface roughening in fluid invasion of porous media.

\footnotetext{
${ }^{\text {a)} E l e c t r o n i c ~ m a i l: ~ j a u m e @ e c m . u b . e s ~}$
}

\section{INTRODUCTION}

The study of the dynamics of an interface between two viscous fluids in the confined geometry of a Hele-Shaw cell, ${ }^{1}$ leading to the phenomenon of viscous fingering, has been studied in great detail from different points of view for more than four decades. ${ }^{2-7}$ While engineers, who first introduced the term viscous fingering, were interested in the so-called secondary oil recovery from porous rocks, ${ }^{8}$ physicists became interested from a more fundamental point of view identifying the problem as a paradigm of morphological instability leading to interfacial pattern formation. ${ }^{9-14}$ Mathematicians have also paid much attention to the also called Hele-Shaw problem as a relatively simple and sometimes solvable free-boundary problem. ${ }^{6,15-20}$ Powerful analytical techniques have also exploited the conformal mapping approach (see some recent examples in Refs. 21 and 22).

The truly seminal work which originated much of these studies is that of Saffman and Taylor in $1958 .^{2}$ The problem of viscous fingering in a rectangular Hele-Shaw cell is often named after them. While clarifying the nature of the instability of a fluid displacing a more viscous one, together with Chuoke, ${ }^{23}$ in complete analogy with the Mullins-Sekerka ${ }^{24}$ instability in solidification, they also posed the so-called selection problem. This is the analogue of the Ivantsov problem for the needle crystal solution of free dendritic solidification. ${ }^{25}$ The full understanding and solution of this selection problem, that is, of the mechanism by which a continuous degeneracy of solutions in the absence of surface tension is reduced to a single observable one, was achieved three decades later, with contributions of several authors. ${ }^{26-29}$ The selection scenario that emerged from these works, sometimes referred to as "microscopic solvability," has become a paradigm of pattern selection in a variety of systems, including most remarkably free dendritic 
growth, ${ }^{12,13}$ and has been highlighted as an important breakthrough in the field of pattern formation in general. ${ }^{14}$

In the last decade the study of Hele-Shaw flows has slightly drifted towards more mathematically oriented research. However, deep and fundamental questions have continued to captivate the interest of physicists. In the context of Laplacian growth, for instance, important progress has been made towards an understanding of the generation of the anomalous dimensions of fractal growth. ${ }^{30-36}$ The understanding of the anomalous exponents characterizing Laplacian growth has escaped a controlled renormalization group handling though, since the problem has an infinite upper critical dimension. This point, and the very concept of universality classes in interface growth, remain as some of the outstanding challenges in nonequilibrium physics, but we will not pursue this line here. On the other hand, some intriguing formal connections to apparently unrelated fields of physics have been reported, for instance in string theory ${ }^{37}$ or in physics in the quantum Hall regime. ${ }^{38}$ These seem to confirm that the potential for surprise ${ }^{37}$ in this apparently simple problem refuses to be exhausted. Even the most traditional version of viscous fingering continues to yield surprising results and new insights for instance in the recent findings in the study of formation of fjords in radial growth. ${ }^{39,40}$

One of the most remarkable results of the last decade in Hele-Shaw flows is due to Tanveer and co-workers ${ }^{18,41,42}$ and has opened a new way of looking at the dynamics of interface dynamics. It basically states that, in general, the limit of vanishing surface tension does not converge to the exact zero-surface tension solutions after a time of order unity, even for exact solutions that behave smoothly, without any finite-time singularity. This means that there is a time which is essentially independent of surface tension, after which the solution with and without surface tension do not approach each other in the limit of vanishing surface tension. This remarkable result and its consequences have been scrutinized recently in the context of a dynamical systems approach ${ }^{7,43,44}$ to viscous fingering, with quite surprising results. ${ }^{45,46} \mathrm{We}$ will briefly review those with the aim at clarifying the role of surface tension as a singular perturbation in fingering dynamics in Secs. IV and V.

Another line of surprises of viscous fingering appear in the study of fluids with similar viscosities. The old conjecture that the basin of attraction of the Saffman-Taylor (ST) finger was indeed dependent on the viscosity contrast, ${ }^{47,48}$ has been put to the test recently, giving rise also to a new view on the problem. We will present these results in Sec. VI. The study of low viscosity contrast flows has also become particularly interesting in the case of rotating HeleShaw cells, where the possible existence of spontaneous interface pinch-off and the importance of wetting effects on the morphology of the resulting patterns has also been remarked, as briefly discussed also in Sec. VI.

The main reason why the problem of viscous fingering has become so popular for so many different purposes is its relative simplicity, both theoretical and experimental. For our purposes here, we would like to emphasize that it is particularly instrumental in yielding new insights in a class of problems for which an analytical handle is usually too difficult.
Within this spirit, one can remark that the Hele-Shaw flows and modifications of them have been used also as model systems to explore more difficult or less controlled situations (see, for instance, Ref. 49). A recent and very illustrative example of this is the use of a modified Hele-Shaw cell with quenched disorder to elucidate longstanding and controversial problems such as kinetic roughening in fluid invasion of porous media. ${ }^{50}$

\section{FORMULATION OF THE PROBLEM}

We consider a Hele-Shaw cell of width $W$ in the $y$ direction and infinite length in the $x$ direction, with a small gap $b$ between the plates. Fluid 1 is injected at $x \rightarrow-\infty$ at a constant velocity $V_{\infty}$ and displaces fluid 2 , and gravity is directed in the $-x$ direction. The fluid flow in this system is effectively two-dimensional and the velocity $\mathbf{u}$ obeys Darcy's law ${ }^{2}$

$$
\mathbf{u}=-\frac{b^{2}}{12 \mu}(\nabla p+\rho g \hat{\mathbf{x}}),
$$

where $p$ is the fluid pressure, $\mu$ is the viscosity, $\rho$ is the fluid density, and $g$ is gravity. Assuming that the fluids are incompressible $(\nabla \cdot \mathbf{u}=0)$ we obtain that the pressure satisfies Laplace equation in the bulk

$$
\nabla^{2} p=0 .
$$

This must be supplemented with the boundary conditions at infinity and at the interface. At infinity, the pressure satisfies

$$
\left.\frac{\partial p}{\partial x}\right|_{|x| \rightarrow \infty}=-\frac{12 \mu}{b^{2}} V_{\infty}-\rho g,
$$

and at the interface, imposing the continuity of the normal velocities yields

$$
\frac{\partial p_{1}}{\partial n}+\rho_{1} g \hat{\mathbf{n}} \cdot \hat{\mathbf{x}}=\frac{\partial p_{2}}{\partial n}+\rho_{2} g \hat{\mathbf{n}} \cdot \hat{\mathbf{x}},
$$

where $\hat{\mathbf{n}}$ is the unit vector normal to the interface. The last boundary condition, in the simplest and more traditional form, is given by the Young-Laplace pressure jump at the interface due to local equilibrium

$$
p_{1}-p_{2}=\sigma \kappa .
$$

$\sigma$ stands for surface tension and $\kappa$ for curvature. This boundary condition is known to be oversimplified for some purposes as we will briefly comment in Sec. VIC. These equations are made dimensionless using $W / 2 \pi$ to scale lengths and the combination

$$
U_{*}=c V_{\infty}+g \frac{b^{2}\left(\rho_{2}-\rho_{1}\right)}{12\left(\mu_{1}+\mu_{2}\right)},
$$

to scale velocities, ${ }^{51}$ where $c$ is the viscosity contrast or Atwood ratio defined as

$$
c=\frac{\mu_{2}-\mu_{1}}{\mu_{2}+\mu_{1}} .
$$

After this scaling the dynamics is controlled by only two dimensionless parameters: The dimensionless surface tension $B$ given by 


$$
B=\frac{\pi^{2} b^{2} \sigma}{3 W^{2}\left(\mu_{1}+\mu_{2}\right) U_{*}},
$$

and the viscosity contrast $c$.

The problem can also be formulated by means of the stream function $\Psi$, the harmonic conjugate of the pressure. ${ }^{51}$ It satisfies $\Delta \Psi=-\Gamma$ where $\Gamma$ is the vorticity distribution, singular and localized on the interface: $\Gamma(\mathbf{r})=\gamma(s) \delta[\mathbf{r}$ $-\mathbf{r}(s)]$. The interface is parameterized with the arclength $s$, and the (dimensionless) vorticity $\gamma$ reads $^{51}$

$$
\gamma=2 c \mathbf{w} \cdot \hat{\mathbf{s}}+2 \hat{\mathbf{x}} \cdot \hat{\mathbf{s}}+2 B \partial_{s} \kappa .
$$

In two dimensions the velocity $\mathbf{w}$ of the interface due to a vortex sheet is given by Birkhoff integral formula

$$
\mathbf{w}=\mathbf{w}(s, t)=\frac{1}{2 \pi} \mathcal{P} \int d s^{\prime} \frac{\hat{\mathbf{z}} \times\left[\mathbf{r}(s, t)-\mathbf{r}\left(s^{\prime}, t\right)\right]}{\left|\mathbf{r}(s, t)-\mathbf{r}\left(s^{\prime}, t\right)\right|^{2}} \gamma\left(s^{\prime}, t\right),
$$

where $\mathcal{P}$ indicates Cauchy's principal value. Equation (10) only accounts for the rotational part of the velocity, w, the one induced by the vortex sheet. In general, the velocity of the interface has also a contribution $\mathbf{u}_{\text {pot }}$ from a potential velocity field, in our case $V_{\infty} \hat{\mathbf{x}}$, so that the velocity of the interface is $\mathbf{u}=\mathbf{u}_{\text {pot }}+\mathbf{w}$. In the particular case of an inviscid fluid displacing a viscous one $(c=1)$ explicit timedependent solutions of the above equations are known for $B=0 .{ }^{15,52,53}$ These solutions are a valuable instrument to understand the subtle role of surface tension in the dynamics. ${ }^{44-46}$

The introduction of a viscosity contrast different from one makes the problem far more difficult to study both from a theoretical and an experimental point of view. For the twosided Hele-Shaw flows, with $c \neq 1$ (referred to as Muskat problem in the mathematics literature) the wide classes of exact solutions for $c=1$ (and $B=0$ ) are not available, and the basic available tool left to study the fully nonlinear regime is numerical computation. The reason for this increased difficulty of the $c \neq 1$ case in comparison to $c=1$ is that, for arbitrary $c$, the two fluids are coupled, while for $c=1$ the pressure of the fluid of negligible viscosity is constant and the pressure of the viscous fluid is formally decoupled from the other one.

In our numerical computations we employ the method introduced by Hou et al. ${ }^{54}$ and used in other numerical studies of the dynamics of Hele-Shaw flows. ${ }^{41,42,45,55,56}$ The method is described in detail in Ref. 54. It is a boundary integral method in which the interface is parameterized at equally spaced points by means of an equal-arclength variable $\alpha$. Thus, if $s(\alpha, t)$ measures arc length along the interface then the quantity $s_{\alpha}(\alpha, t)$ is independent of $\alpha$ and depends only on time. The interface is described using the tangent angle $\theta(\alpha, t)$ and the interface length $L(t)$, and these are the dynamical variables instead of the interface $x$ and $y$ positions. The evolution equations are written in terms of $\theta(\alpha, t)$ and $L(t)$ in such a way that the high-order terms, which are responsible of the numerical stiffness of the equations, appear linearly and with constant coefficients. This fact is exploited in the construction of an efficient numerical method, i.e., one that has no time step constraint associated with the surface tension term yet is explicit in Fourier space. We have used a linear propagator method that is second order in time, combined with a spectrally accurate spatial discretization.

\section{BEYOND THE SAFFMAN-TAYLOR INSTABILITY}

\section{A. Weakly nonlinear analysis of fingering patterns}

The morphological instability of the interface between a less viscous fluid displacing a more viscous one under the conditions of Darcy flow, such as in a porous medium or a Hele-Shaw cell, the so-called ST instability was established in Refs. 2 and 23. Since then, most efforts have concentrated in the so-called steady state selection problem. This subtle and deep problem has to do with the singular perturbation nature associated to the surface tension parameter and has now become a prototype example of the so-called asymptotics beyond all orders. ${ }^{57}$ Most analytical progress has been possible only in the limit of small surface tension. In a series of recent papers, however, it has been pointed out that concerning the dynamics of the early nonlinear stages and for the process of finger competition in finger arrays, the dimensionless surface tension must be considered to all effects as a quantity of order one. ${ }^{58,59}$ That is, the typical scale of the interface morphology which results from the linear ST instability is necessarily that in which the stabilizing forces of surface tension are of the same order than the destabilizing viscous forces. With the intention to have an analytical handle which was not perturbative in any of the two parameters of the problem, we introduced a weakly nonlinear expansion of the dynamical equations in Refs. 58 and 59. This allows one to extend the linear dynamics into the weakly nonlinear regime in a systematic way and to elucidate the interplay between the distinct parameters and the different nonlinearities.

The basic idea is to define a formal gradient expansion (in a nonlocal problem) in terms of a book-keeping parameter $\epsilon$ as a ratio of the scale of variation of the height $h(x, t)$ of the interface to a horizontal scale $L$ of the form

$$
\frac{\partial h}{\partial t}=F[h]+\epsilon G_{2}[h]+\epsilon^{2} H_{3}[h]+\ldots,
$$

where $F[h]$ is a linear (nonlocal) operator, $G_{2}[h]$ a quadratic one, etc. Apart from useful insights into the interplay between parameters, geometry and nonlinearity (see an application in Sec. VI A), this formalism provided a systematic way to obtain the different nonlinear orders of the nonlocal interface equation relevant to renormalization group studies of kinetic roughening in Hele-Shaw flows subject to quenched disorder. $^{50}$

\section{B. Nonlinear Saffman-Taylor instability}

In close connection with this analysis, an exact result has been reported which uncovers the full nonlinear structure of the ST instability, as a subcritical bifurcation. ${ }^{60}$ It has been shown that, for any given set of parameters, one can find modes that are linearly stable but nonlinearly unstable, that is, that finite-sized perturbations may grow unstable if they 


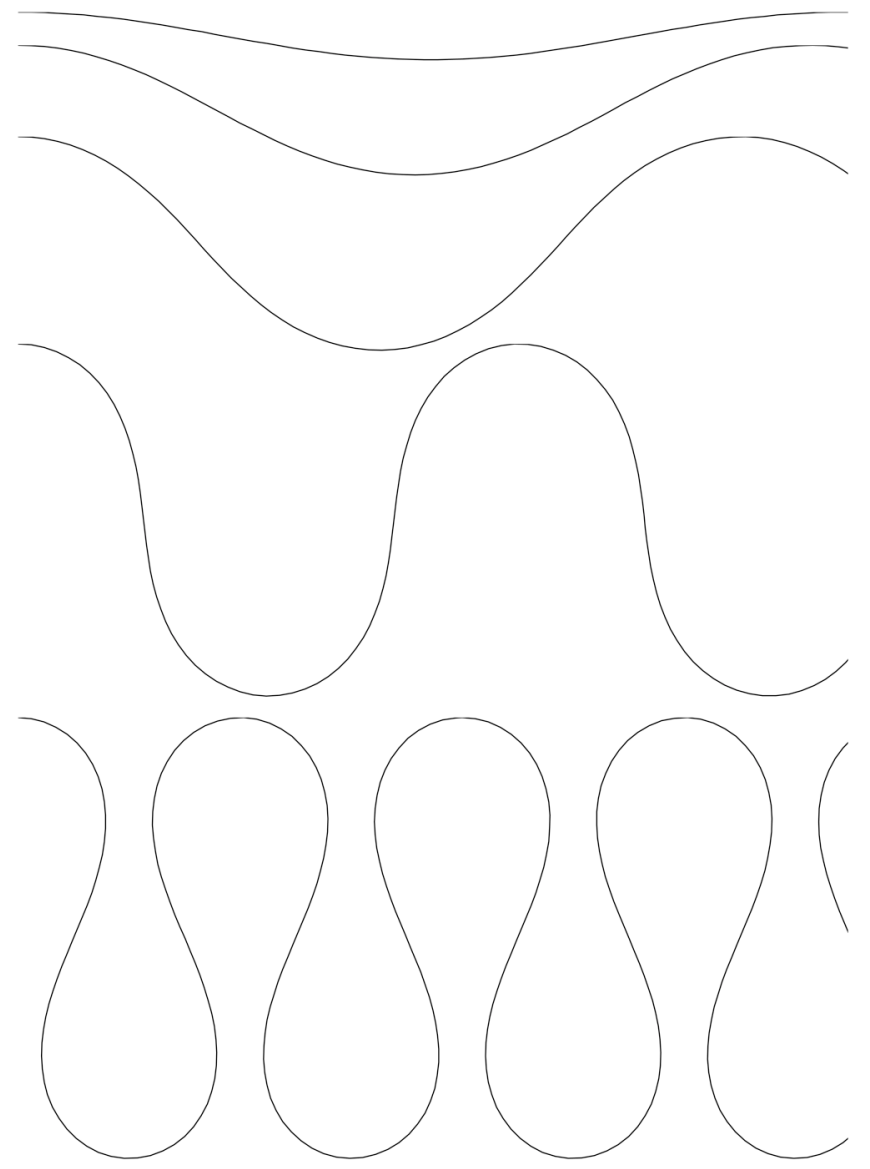

FIG. 1. Sample of exact stationary solutions of the ST problem with finite surface tension (elastica solutions) which balance capillary with viscous forces. Each interface configuration is a point in the subcritical branch of the nonlinear ST instability.

surpass a certain amplitude threshold. The latter has been determined exactly and has been identified as a branch of exact solutions that bifurcate from the instability and ends up at a topological singularity. These solutions had been reported before ${ }^{61}$ in a different context and have a clear physical interpretation. They consist of interface shapes which balance exactly capillary and viscous forces, yielding a family of solutions which coincided, in the case of channel geometry, with the so-called elastica solutions of ideal rods. The precise prediction of the amplitude-threshold associated to these results has been checked directly in experiments using a new experimental procedure to prepare initial conditions à la carte for the interface shape in a Hele-Shaw cell. ${ }^{60}$ Examples of such solutions are shown in Fig. 1. Incidentally, the above procedure to prepare arbitrary initial conditions for the interface shape in a Hele-Shaw experiment does open new and interesting possibilities to study transient dynamics.

\section{DYNAMIC SOLVABILITY}

\section{A. Extending solvability theory}

The understanding of the delicate role of surface tension as a singular perturbation yielding an effect "beyond all orders" has been celebrated as a major achievement in pattern formation. This surprising and indeed generic mechanism of steady state selection in interfacial growth problems is some- times referred to as "microscopic solvability." 10 The term "microscopic" is inherited from the context of solidification growth, due to the fact that it is the presence of a microscopic length scale (the capillary length) what is crucial in fixing the macroscopic scale of the dendrite.

Once this problem was understood, a new generation of results started to arise concerning the dynamics of HeleShaw flows. On the one hand, it was pointed out that the picture of finger competition based on the idea of simple Laplacian screening to explain the evolution towards the single finger solution was too naive at least in two respects: First, for viscosity contrasts not very high, the dynamics was observed to be qualitatively different, ${ }^{47,48}$ and second, the role of surface tension on the dynamics was recognized as far from being trivial. We will address the effects of viscosity contrast in Sec. VI. Here we now concentrate on the singular effects of a small surface tension.

Among the results that triggered most interest in studying the nonlinear dynamics of viscous fingering at an analytical level, we can remark the obtention of explicit, exact time-dependent solutions of the problem for zero surface tension, thanks to the use of conformal mapping techniques. ${ }^{15,16,19,53,62}$ Remarkably, the classes of solutions found were very broad and included also large classes of solutions behaving smoothly, that is, not leading to finitetime singularities of any type. The rare and remarkable fact of having exact time-dependent solutions of a strongly nonlinear and nonlocal free-boundary problem was indeed a good opportunity to gain analytical insight into interfacial dynamics in general. The natural question then arose as to whether a selection mechanism, analogous to that which works for the statics (selection of a stationary single finger), could be defined for the dynamics. That is, whether one could conceive a dynamical solvability scenario associated to surface tension. Note that the situation was indeed completely parallel to the steady state selection problem, that is: (i) One neglects surface tension; (ii) one solves the problem and finds it is degenerate and, in a sense, unphysical; (iii) one restores surface tension to show that the degeneracy is broken and that the physical solution survives. The idea is that, although the physical problem is not degenerate and, therefore, the "selection" problem is actually introduced by the removal of a crucial physical ingredient, through this procedure an important insight was gained into the mathematical and physical nature of the problem. It could then be interesting to follow a similar scheme for the dynamics and try to learn something from the mathematical nature of the problem by following a similar procedure. This was in principle possible because the exact solutions were available. The pursuit of this idea originated a series of papers ${ }^{43-45,63,64}$ which we will summarize in the following sections. The need to define and compare different types of dynamics lead us to adopt a dynamical systems point of view, which deals basically with global (topological) properties of ensembles of trajectories in phase space rather than looking at specific trajectories. ${ }^{65}$ 

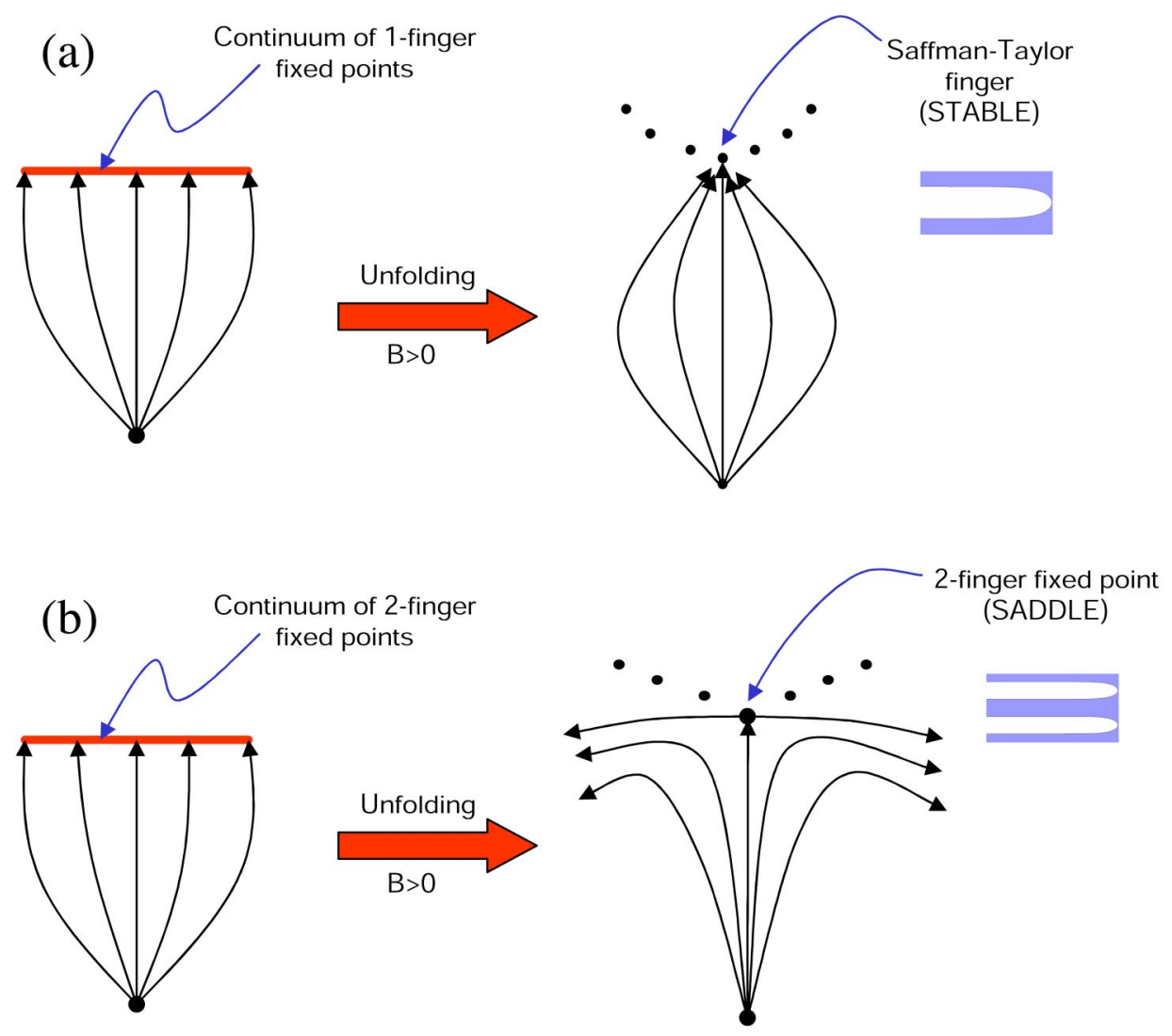

FIG. 2. (Color online). Schematic representation of the unfolding of nonhyperbolic fixed points into hyperbolic ones by the introduction of surface tension, in the case of single-finger and two-finger selection.

\section{B. Multifinger selection: A dynamical systems approach}

A first step in this direction was to try to generalize the steady state selection theory of the single finger solution to the case of multifinger solutions. ${ }^{64}$ It was indeed known that steady state solutions with coexisting unequal fingers existed for zero surface tension, while only the symmetry degenerate case of equal fingers could seem a priori physically acceptable. Furthermore, there was a continuum degeneracy of such multifinger solutions so it seemed natural to try to extend solvability theory to these cases. This was indeed accomplished explicitly for two-finger configurations in a channel. ${ }^{64}$ We showed that by a mechanism similar to that of the single-finger solvability, the correct introduction of surface tension collapsed the two-parameter continuous family of two-finger solutions into a discrete set of solutions described by two integer indices. Those solutions were supposed to be unstable except for one, corresponding to the symmetry-degenerate double ST finger, which had a saddle point structure, that is, with some stable directions (inherited from the single finger stability) and some unstable directions.

In the spirit and language of dynamical systems theory, we can recast the traditional solvability theory and the above extension to the multifinger case in the following way. The phase space flow emerging from the planar interface unstable fixed point, in the absence of surface tension, ends up at a continuum of fixed points. This yields a nonhyperbolic flow, since the line of fixed points is marginally stable. The introduction of surface tension yields a natural unfolding of this otherwise structurally unstable ${ }^{65}$ flow and restores the hyper- bolicity of the fixed points by isolating a single stable fixed point (the ST finger), while the rest of (now isolated) fixed points are unstable and thus unobservable. Within this context, the extended solvability theory to multifinger configurations can also be interpreted as the restoring the hyperbolicity of the flow through the unfolding provided by surface tension. The crucial difference now is that the solution which is singled out is not the stable fixed point (the global attractor of the problem) but a saddle fixed point, which corresponds to the symmetry-degenerate equal finger solutions. The rest of discrete and isolated fixed points are also unstable. The saddle point structure of the flow is actually a consequence of the stability of the single finger ST solution on the one hand (responsible of the attracting part of the flow) and of the global instability of a periodic finger array, as described by Kessler and Levine, ${ }^{66}$ on the other hand, which is responsible for the phenomenon of finger competition and thus originates the unstable directions of the flow, corresponding to modes that break the symmetry of the equal finger configuration. Figure 2 depicts a schematic representation of this discussion.

An important difference between multifinger solvability theory and the traditional single-finger one, is precisely the saddle-point structure of the selected solution. In this case this has dramatic consequences for the phase space flow structure in regions far away from both the attractor (the stable ST single finger) and the planar interface (unstable) fixed point. That is, by restoring the saddle-point structure of the equal-finger fixed points, the global phase space structure is essentially modified. This is qualitatively different from 


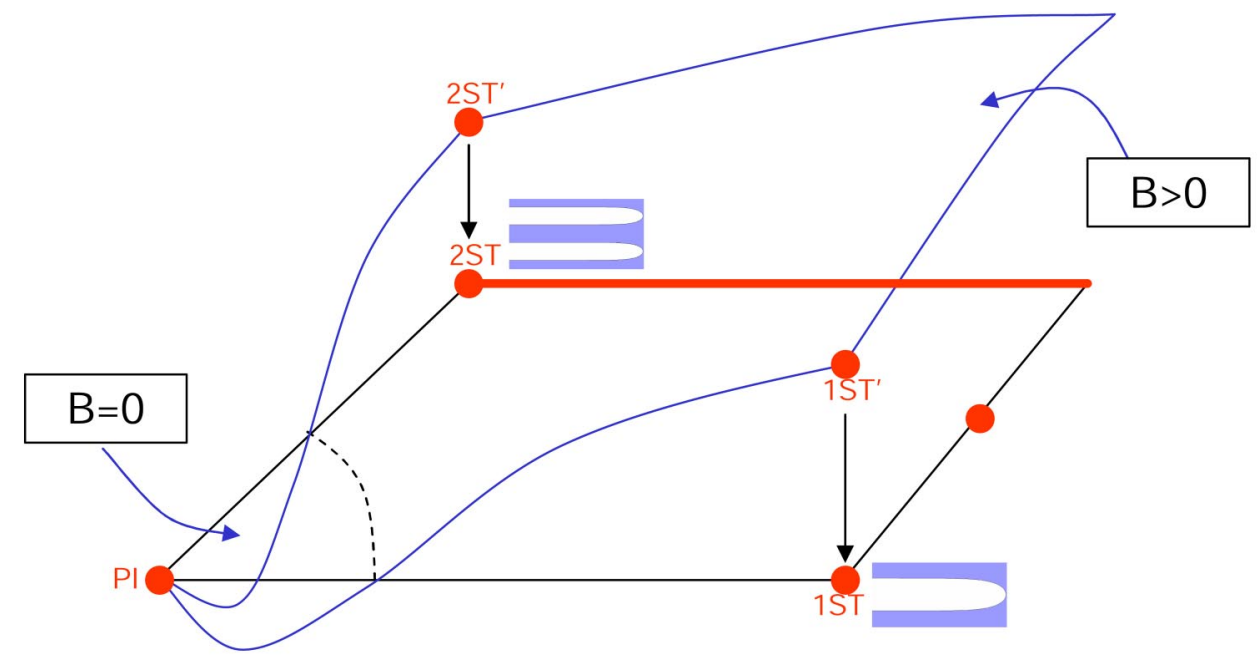

FIG. 3. (Color online). Schematic representation of different twodimensional (2D) dynamical systems embedded in the infinite-dimensional phase space. The flat surface is the zero surface tension dynamics. The curved surface intersects the other by construction in a line of initial conditions, and is defined by the evolution of these with finite surface tension. Single-finger and two-finger fixed points are depicted as 1ST and 2ST, with the prime designating the case with $B \neq 0$.

the static solvability case, in which only the local structure around the attractor is unfolded. In this sense we refer to multifinger selection as dynamic solvability theory. It is not a dynamical selection in the sense that surface tension selects particular sets of trajectories. However, the solvability of the multifinger fixed points corresponds to the unfolding of a structurally unstable flow and, therefore, it transforms the global (topological) structure of phase space. In the next section we will pursue this general qualitative picture in more detail to reach a more quantitative scenario and to assess to what extent trajectories evolving with and without surface tension can depart from each other even if reaching the same final attractor.

\section{SINGULAR EFFECTS OF SURFACE TENSION: BEYOND DYNAMIC SOLVABILITY}

In the preceding description, it has been established that the introduction of a vanishingly small surface tension has a strong impact on the global structure of the phase space flow. The idea that the flow with and without surface tension was not topologically equivalent was already recognized in Ref. 43 with the study of the simplest exact solutions. However, one could hope for making a step further and have a more detailed description of the effect of surface tension on the trajectories in phase space. Our proposal was then to focus on specific low-dimensional invariant subspaces of the dynamics, taking advantage of the exact solutions available. These yield sets of ODE's for the finite sets of parameters in the conformal mapping describing the solutions (see more details in Refs. 44-46). The idea is then to look at those sets of ODE's as low dimensional dynamical systems and discuss their properties. A geometrical way to illustrate the comparison between zero-surface tension dynamics and small surface tension dynamics, for two-finger configurations, is shown in Fig. 3. Note that the invariant manifolds of the two different dynamics, when imbedded in the (infinitedimensional) space of interface configurations cannot coincide. We can construct a two-dimensional dynamical system with nonzero surface tension dynamics as the one defined by the evolution to infinite time (forward and backward) starting from a line of initial conditions corresponding to the class which is solvable for the zero surface tension dynamics. The two surfaces defining two different dynamical systems of the same dimensionality do intersect, by construction, in one line. They also have in common the planar interface fixed point. If we now take the limit of vanishing surface tension we can ask in which way the two surfaces will approach each other. It is clear that, consistently with solvability theory, both single finger and two-finger fixed points with finite surface tension will approach the corresponding solutions with zero surface tension. Since the flow topology cannot be the same in the two cases, it is necessary to study more systematically the effect of surface tension in specific trajectories. This can be accomplished thanks to the asymptotic theory developed by Tanveer and co-workers, and with the help of numerical computation.

\section{A. Asymptotic theory}

The starting point of the perturbative framework developed by Tanveer ${ }^{18}$ is the fact that the zero-surface tension problem is ill-posed as an initial-value problem. Once the ill-posedness is cured through the proper analytical continuation of the problem, ${ }^{67}$ a well defined perturbative scheme is possible.

The remarkable and surprising result that this analysis unveiled is the fact that, in general, the limit of trajectories for vanishingly small surface tension can only converge to trajectories evolved with zero surface tension for a time of order one, that is, a time that does not diverge as surface tension tends to zero. ${ }^{41,42}$ While this would be an expected result for solutions leading to finite-time singularities in the case of $B=0$, it is not at all intuitive for smooth evolutions of both cases, and even more surprising if the two evolutions evolve towards the same attractor at infinite time, as will be the case. For the linearized (or weakly nonlinear) dynamics of the problem, for instance, the time $T$ for which the $B$ $=0$ and the $B \neq 0$ converge scales as $T \simeq-\log B$. The phenomenon reported by Siegel and Tanveer, thus, concerns the strongly nonlinear dynamics.

The basic idea of Tanveer's asymptotic theory is that one 


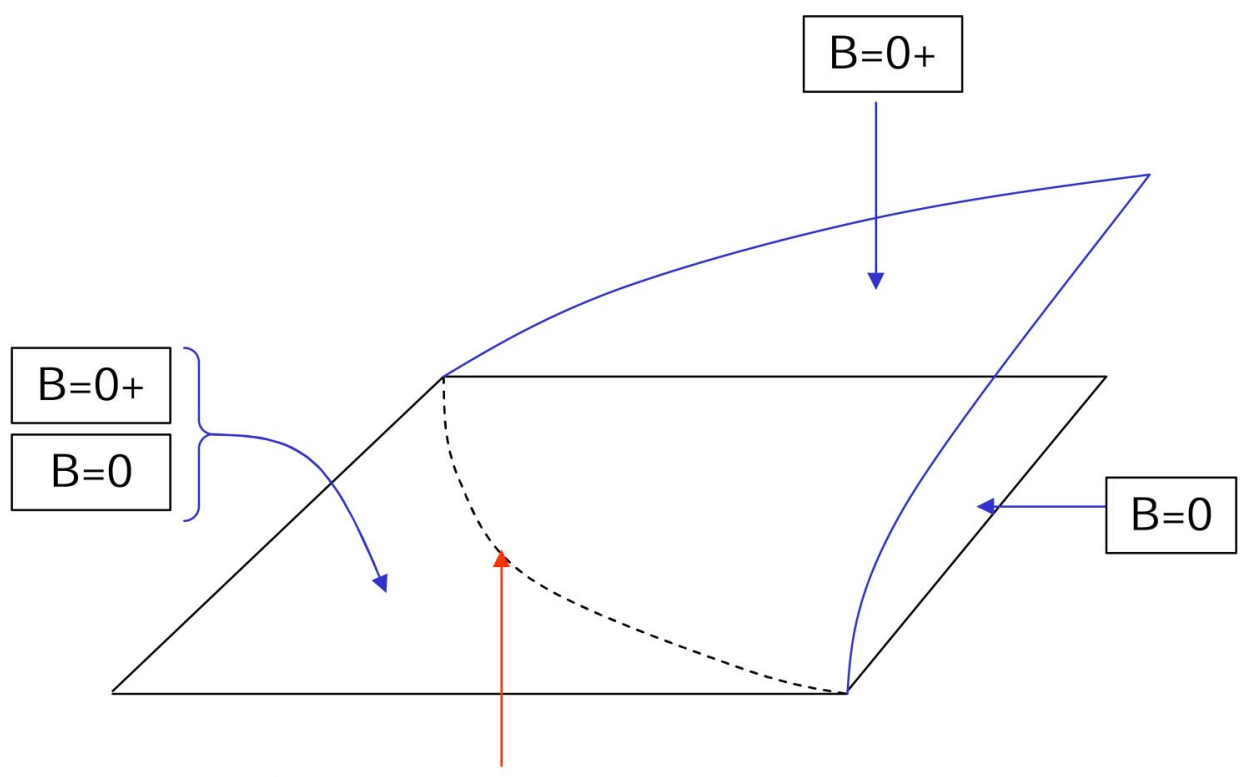

FIG. 4. (Color online). Comparison of the two invariant manifolds of Fig. 3 for $B=0$ and for the limit $B=0^{+}$. The two surfaces coincide until the impact of daughter singularities and separate from each other after that.

Line of impact of daughter singularities

can construct a correction to the zero-surface tension dynamics which depends only on that zeroth order dynamics and which predicts its own failure according to the following scenario. The leading surface tension correction modifies the analytical structure of the conformal mapping which describes the interface shape by giving rise to a localized cloud of the so-called daughter singularities. This cloud has a size of order $B^{1 / 3}$ and its evolution is determined once the zeroth order is solved. Generically, one of these daughter singularities may hit the unit circle (which is mapped to the interface) within a finite time. This then signals the failure of the perturbation scheme. For times after this event, the two dynamics may differ significantly.

\section{B. Numerical results and general picture}

Tanveer and co-workers checked this scenario with numerical simulations in the case of single-finger evolutions. ${ }^{41,42}$ For instance, they showed how the evolution with $B=0$ of the interface leading to a finger with the wrong width (different from the $1 / 2$ relative size predicted by selection theory), was indeed correct until a finite time well into the strongly nonlinear regime (with a well developed finger). After that time, identified by the impact of a daughter singularity, the dynamics with small but finite surface tension abruptly modified the evolution towards the correct finger width.

The challenge was then to check this scenario in situations where different fingers coexist and compete. A comprehensive study of these situations for very small surface tension is very demanding numerically and was performed in Refs. 45 and 46. The most interesting configurations studied were those in which the competing fingers were consistent with the correct final width selected by surface tension. In these cases, the dynamical role of surface tension could be isolated from the selection phenomenon itself. While the picture of the asymptotic theory was checked to be consistent, a series of quite surprising results were found.
The most striking result was the observation that nonzero measure sets of initial conditions corresponding to unequal fingers could be found for which the evolution with zero surface tension would give the wrong output of finger competition. That is, in a configuration with two unequal fingers, the one approaching the single finger stationary solution (the winner) would be different for zero surface tension than for any arbitrary value of surface tension, no matter how small. Therefore, the attracting fixed point for $B=0$ and for $B=0^{+}$do coincide, but the path in phase space to reach this attractor is completely different. Notice that this is an intrinsically dynamic phenomenon, unrelated to the steady state selection problem, since the width of the selected finger at the end is the same in both cases. Remarkably, this surprising behavior is encountered in a finite measure region of phase space. ${ }^{45,46}$ The modification of the phase space flow structure by surface tension is thus dramatically manifest in this region.

In Refs. 45 and 46 it is shown in explicit examples how the impact of the daughter singularity signals the time after which the evolution with $B=0$ differs significantly form that of small but finite surface tension. From this scenario, then, we can conclude that the trajectories with finite surface tension converge one to one to those for $B=0$ for a finite time (which can be evaluated in terms of the $B=0$ dynamics). The resulting picture in the limit of $B=0^{+}$can thus be illustrated schematically as in Fig. 4. This results from the construction of Fig. 2, taking the limit of the line of intersection of initial conditions to approach the planar interface fixed point, and then taking the limit $B \rightarrow 0$. Then, the two surfaces describing the two dynamical systems will coincide in a finite region containing, in addition to the planar interface fixed point, the single-finger and the doubly degenerate (saddle) fixed point. This region will end at the line of impact of the daughter singularities. The subsequent evolution in both cases will differ then, and thus the surfaces $B=0$ and $B=0^{+}$do split beyond that line. 
The general conclusion is thus that, for a time of order one (which depends on the specific initial condition but can be determined exactly from the zero-surface tension dynamics) surface tension behaves as a regular perturbation and the effect of introducing a small value of $B$ is small. After that time, the evolution is not only quantitatively different but also qualitatively, since the topology of the phase space flow is different. While it is not ruled out that some initial conditions do not differ qualitatively, it can also occur that in some finite ranges of initial conditions the trajectories with the two dynamics depart completely from each other, even if they eventually approach the same final attractor.

\section{Noise and the limit of small surface tension}

In the above discussion we have been dealing with the role of surface tension as a singular perturbation in the dynamics corresponding to the high viscosity contrast limit. In this case, it has been commonly believed that, in the channel geometry, the single-finger ST solution is the universal attractor of the dynamics. However, in the case of very small surface tension this view has been questioned on the basis of the effect of noise, stating that for very small surface tension the behavior of the system is essentially chaotic. ${ }^{68}$ The argument is based on the instability of fingers to tip-splitting when surface tension is small. In this respect it is worth clarifying that the tip-splittings observed in simulations such as those of Ref. 68 are spurious effects of round-off noise which can be suppressed by a proper noise filtering scheme. The difficulty lies in the fact that the sensitivity to the noise is enhanced as surface tension is decreased. This makes the numerical simulation of small surface tension flows very demanding. A careful analysis clearly shows that once the noise is appropriately filtered, however, the intrinsic dynamics does lead the interface to the ST finger, no matter how far from it. The conclusion that this is the universal attractor just needs to be taken prudently if there is a finite amount of noise. Then if one takes the limit of small surface tension, for a fixed amount of noise, one would certainly reach a point where the ST finger is not stable to finite perturbations and then the chaotic picture described in Ref. 68 would be relevant. This issue is thus only a matter of noncommuting limits (small surface tension vs weak noise). The intrinsic dynamics of the free boundary problem as defined (in the absence of noise), however, does have a unique and universal attractor, namely the ST finger.

This last statement refers to the case of high viscosity contrast limit $c=1$. However, in the following section we will see that the dynamics is much reacher and puzzling once the viscosity contrast, the other dimensionless parameter of the problem, is allowed to depart from that limiting value. Then, the ST attractor has only a finite basin of attraction, even for surface tension of order one, and new physics is found. In this case, however, this phenomenon is intrinsic to the dynamics and has nothing to do with the noise sensitivity just discussed above.

\section{DYNAMICS OF FINGER COMPETITION: THE ROLE OF VISCOSITY CONTRAST}

The general picture of finger competition where fingers try to screen the Laplacian pressure field from each other ending up at a single finger surviving and approaching the ST solution, even including the singular effects of surface tension, is restricted to the one-sided Saffman-Taylor problem, that is, to viscosity contrast $c=1$ where $c=\left(\mu_{2}\right.$ $\left.-\mu_{1}\right) /\left(\mu_{2}+\mu_{1}\right)$. Already in the 1980s, Tryggvason and Aref $^{51}$ observed numerically that the viscosity contrast has a strong influence on the dynamics of Hele-Shaw flows and consequently in the morphology of the fingering patterns formed. Their analysis was rather qualitative though, due to some extent to the computer limitations of that time. This numerical evidence was later confirmed by the experimental results obtained by Maher ${ }^{69}$ using a experimental setup where the instability was driven by gravity and the fluid used was the binary-liquid mixture isobutyric acid plus water at critical composition, that allowed to reach very low values of the viscosity contrast parameter. Simple (two finger) configurations were also studied ${ }^{70}$ by means of direct numerical integration that confirmed the dramatic differences between high $c$ and low $c$ dynamics. The conclusion was that for low viscosity contrast the finger competition process was strongly inhibited, and the coarsening process observed for high viscosity contrast ${ }^{71,72}$ that leads to the formation of a single finger does not seemed to take place.

In an attempt to clarify the issue on more rigorous grounds, in Refs. 47 and 48 we developed a topological approach to study finger competition that allowed for a different view and new insights on the dynamics of low $c$. We conjectured that the size of the basin of attraction of the Saffman-Taylor depended on the value of $c$. That is, even though a ST finger solution exists and is stable for any value of $c$, it might not be the universal attractor of the dynamics for any viscosity contrast. Obviously this raises the question about the long time fate of the system when not attracted to a single finger configuration. With the current computer power and the substantial progress made on the numerical algorithms for this kind of problems, it seemed thus appropriate to reconsider those open questions and try to shed new light into the problem, both testing the scenario conjectured in Refs. 47 and 48 and providing a more quantitative characterization of the sensitivity to viscosity contrast. In addition, there is another fundamental reason to explore this issue with precise numerics, that is, the relevance to the fundamental question on the occurrence of topological singularities associated to interface breakup through pinch-off. ${ }^{73}$ For low viscosity contrast, indeed, one observes both in experiments and simulations, an enhanced tendency to interface pinchoff. While we will not specifically address here the nontrivial question of whether the dynamics leads spontaneously to finite-time pinch-off, we will push the idea that the tendency to pinch-off can be related to the fact that attractors with different topology coexist and compete. Recently, the problem of Hele-Shaw flows with arbitrary viscosity contrast, largely neglected in the literature in comparison to the high viscosity case, has received some attention also from a mathematical point of view. Howison ${ }^{20}$ has presented a formal 
technique for finding explicit solutions to the two-phase flow in a Hele-Shaw cell, with the confessed intention to drive the attention of the community to this fundamental problem.

\section{A. Basin of attraction of the Saffman-Taylor finger}

The aim of the present subsection is to present a first attempt to confirm and characterize in a quantitative way the dependence of the basin of attraction of the ST finger on viscosity contrast $c .{ }^{46,74}$ Obviously, the numerical exploration of the infinite-dimensional phase space of the problem can only be undertaken partially. It is thus crucial to devise an optimal strategy in selecting the class of interface configurations which will be most useful to elucidate the generic questions posed on the dynamics with a minimal numerical effort.

It seems clear that two-finger configurations will be adequate to study finger competition. We will choose initial conditions with two sinusoidal modes, with wave numbers $k$ and $2 k$, with small amplitudes so that their growth is initially linear. Furthermore, we will first choose surface tension in such a way that the two modes have exactly the same linear growth rate. This is always possible and has the great advantage that the ratio between the two mode amplitudes is kept constant as long as the dynamics is linear. Deviations from this constant ratio will directly signal nonlinear interactions. In addition, with this condition the linear growth yields a selfsimilar solution and the actual initial amplitude of the modes is thus an irrelevant parameter. The amplitude ratio is then the only parameter that spans the phase space. This one-dimensional projection is obviously a drastic simplification but we will see that it provides useful insights. It can obviously be made less restrictive a posteriori to assess the range of validity of the partial conclusions. In any case we will deal with dimensionless surface tension of order unity $(B \approx 1)$. Note that, as stated above, this range of values is the relevant one for configurations of fingers emerging spontaneously from the linear instability of the planar interface, since they occur precisely at the scale where capillary and viscous forces are of the same order. We will not address here the small surface tension limit for general viscosity contrast, although we recognize this is a interesting and completely open problem. We will briefly comment on that in Sec. VII.

The simplest initial condition to study finger competition is thus a two-finger (or two-bump) interface, with two Fourier modes of the form

$$
x(y)=-a_{1} \cos (y)+a_{2} \cos (2 y),
$$

where both $a_{1}$ and $a_{2}$ are real and positive. The form Eq. (12) describes an interface with one or two bumps, depending on the ratio $a_{1} / a_{2}$ : If $a_{1}<4 a_{2}$ the interface has two bumps, and one otherwise. The values of $a_{1}$ and $a_{2}$ are chosen small enough to guarantee that the initial interface is well inside the linear regime. The two modes present in Eq. (12) have equal growth rates for a surface tension value $B=1 / 7$.

It is important to stress at this point that the evolution in the case where any of the two amplitudes is zero leads to the ST finger (single or double), regardless of viscosity contrast. The basin of attraction of the ST is thus always finite. The
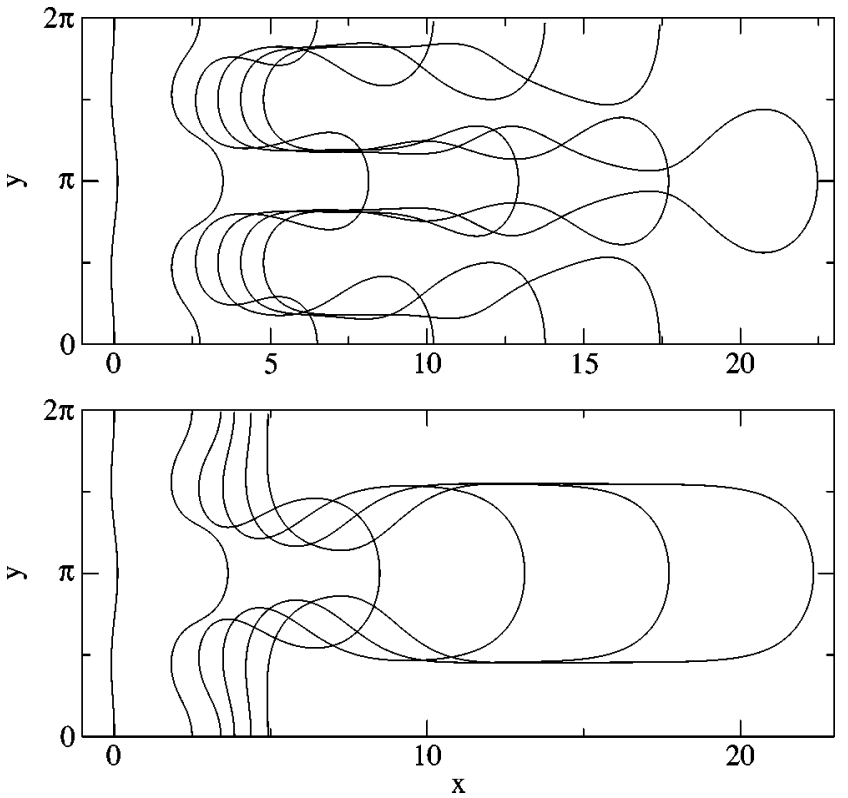

FIG. 5. Evolution of the initial condition $a_{1}=0.05$ and $a_{2}=0.07285$, with $B=1 / 7$ and $c=0.0$ (upper plot) and $c=0.8$ (lower plot).

intrinsic differences between high and low viscosity contrast refer only to the process of finger competition, that is, they are manifest as long as unequal fingers coexist.

We have numerically computed the evolution of the initial condition Eq. (12) for various values of the viscosity contrast, surface tension and initial conditions. We have observed that for long times the interface exhibits two different kinds of configurations, illustrated in Fig. 5 and consistently with the two types of finger dynamics observed in Refs. 47 and 48 for the two extreme values of $c=1$ and $c=0$. The two types of dynamics give rise to two distinct morphologies as follows. As usually seen for high viscosity contrast, in what we call type I dynamics the leading finger screens out the trailing one by suppressing its growth to the point that the small finger is completely halted or exhibits a residual evolution driven by surface tension. The key defining point of type I dynamics is that the leading finger widens to attain a stationary shape close to the single-finger solution predicted by selection theory, thus absorbing all the injected flux, while the secondary finger is either completely suppressed or frozen.

In the second type of behavior (or type II), which is typical of lower viscosity contrast, the growth of the second finger is not halted, although its speed may decrease a considerable amount with respect to the speed of the large finger. At long times the large finger advances approximately at constant velocity, but with a substantial difference with respect the previous case: The finger sides bend to give rise to a narrow neck behind the leading head. This neck can become extremely narrow to the point of approaching a possible topological singularity in the form of interface pinchoff. The appearance of some sort of a neck is rather usual even for high viscosity contrast, since fingers typically develop overhangs. Indeed, at short times both fingers are substantially narrower than half the channel width, and later in the evolution the region of the leading finger that is ahead 
widens also in type I competition. The key point here, however, is that the narrow neck in type II dynamics supports a vanishingly small flux, so that the leading head or bubble increases its area only very slowly. On the other hand, there is an amount of flux that feeds the secondary finger which then exhibits a nontrivial dynamics which persists in one way or another for all times.

When a pronounced necking develops, the shape of the quasi-bubble formed is very close to the zero surface tension bubble shape described in Ref. 75. The second (smaller) finger exhibits a variety of behaviors: It may or may not develop necking, and it can also present tip-splitting, but in all cases it will exhibit some sort of "persistent" dynamics. Note that the whole system is becoming more and more elongated with time so there is increasing space for the secondary finger to evolve independently of the leading tip region of the primary finger. We have not observed any clear indication of a steady state behavior of the secondary finger in type II dynamics, although the tip region of the leading finger often reaches a practically stationary shape in a reasonably short time. The two morphologies and corresponding dynamics just introduced were described and characterized with topological properties of the fluid flow in Refs. 47 and 48 for the extreme values of $c$. Here we will see that for a given initial condition, the system will display unambiguously one of the two behaviors depending on the viscosity contrast. Remarkably the transition between the two behaviors is quite sharp, with slight changes in the value of the viscosity contrast driving the system from one kind of behavior to the other one.

With the simplest choice of surface tension $B$ described above to assure the same linear growth of the two modes, according to the linear dispersion relation of the problem ${ }^{23}$

$$
\omega(k)=|k|\left(1-B k^{2}\right),
$$

we are left, in our first analysis, with a uniparametric family of self-similar initial conditions in the linear regime. ${ }^{87}$ The quantity we will use to parameterize the two-bump initial condition Eq. (12) is the ratio between the tip difference and the total width of the interface, measured as the length difference between the maximum and the minimum of the interface. This parameter will be called $d$, and for our initial conditions it reads

$$
d=\frac{1}{\frac{1}{2}+\frac{1}{16} \frac{a_{1}}{a_{2}}+\frac{a_{2}}{a_{1}}} .
$$

$d$ is a function of the ratio $a_{1} / a_{2}$, and since the surface tension is chosen to make the growth rate of both $a_{1}$ and $a_{2}$ the same, $d$ remains constant throughout the linear regime. Then, the value of the viscosity contrast at which the transition between type I and type II dynamics takes place depends on the initial condition, or equivalently, is a function of $d$.

As a precise criterion to establish the distinction between types I and II dynamics, we have used the area covered by the small finger and the bottom line of the interface. This definition has no ambiguity for our class of initial conditions, and the results do not depend significantly on the details of such definition. Then, the dynamics is of type I if the area of

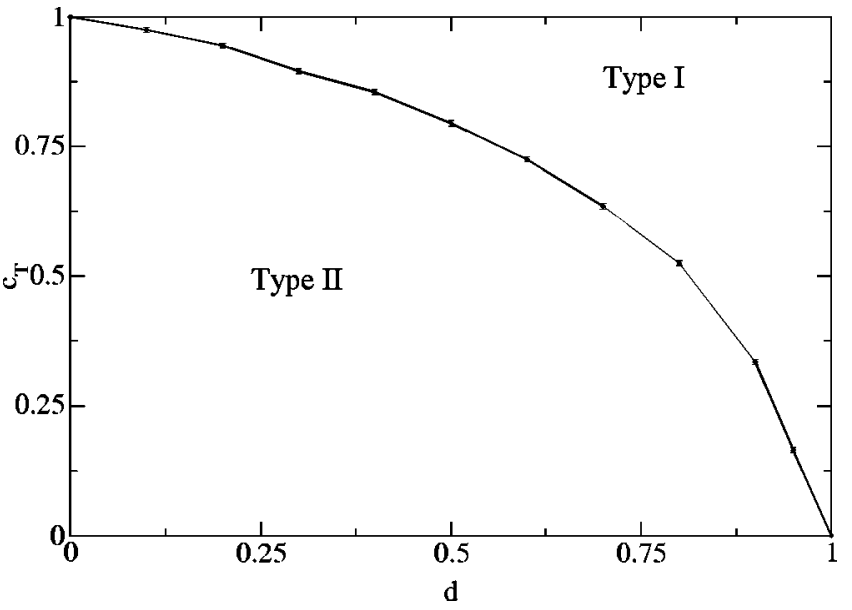

FIG. 6. $c_{T}$ vs $d$, for an initial condition of the form Eq. (12) and surface tension $B=1 / 7$.

the small finger approaches a constant value for long times, and type II otherwise. We have applied this criterion to study systematically the dependence of the viscosity contrast transition value $c_{T}$ on the initial condition. In Fig. $6 c_{T}$ versus $d$ is plotted, for an initial condition of the form Eq. (12) and surface tension $B=1 / 7 . d=0$ corresponds to two equal bumps, and $d-1$ corresponds to a single bump. From the plot it can be seen that, as the lengths of the two initial fingers become close to each other, the viscosity contrast that drives the dynamics into the type I dynamics tends to $c=1$. For $c=1$, regardless of how small the initial difference in finger tip position is, the long time interface configuration consists of a steady Saffman-Taylor finger (type I). In opposition to this limit, when the length of the small finger tends to zero, type I dynamics occurs for any value of the viscosity contrast. Then, if the initial interface consists of a single bump the long time interface will be a Saffman-Taylor finger. The convexity of the curve $c_{T}(d)$ shows that type II dynamics has a larger basin of attraction than type I dynamics. Furthermore, the very small slope of the boundary between the two behaviors in Fig. 6 when approaching $c=1$ is telling us that the maximal sensitivity to viscosity contrast is precisely at $c \approx 1$. The physical picture of finger competition based upon Laplacian screening, which is the common one for the high viscosity contrast case, happens to be less generic than the low contrast behavior. Figure 6 describes the variation and sensitivity of the basin of attraction of the ST finger to viscosity contrast, and tells us, given a value of $c$ and $d$, whether or not the dynamics is attracted to the ST finger.

The weakly nonlinear approach described in Sec. III A and Ref. 58 can be applied to the present problem in order to gain some insight into the dependence of the dynamics on the viscosity contrast at the early nonlinear stages of the evolution. According to the weakly nonlinear equations, the amplitudes $a_{1}(t)$ and $a_{2}(t)$ of the two relevant modes obey the following equations:

$$
\frac{\dot{a}_{1}(t)}{a_{1}(t)}=\frac{6}{7}\left\{1+2 c a_{2}(t)+\left[4 c^{2}-1\right] a_{2}^{2}(t)-\frac{3}{4} a_{1}^{2}(t)\right\},
$$




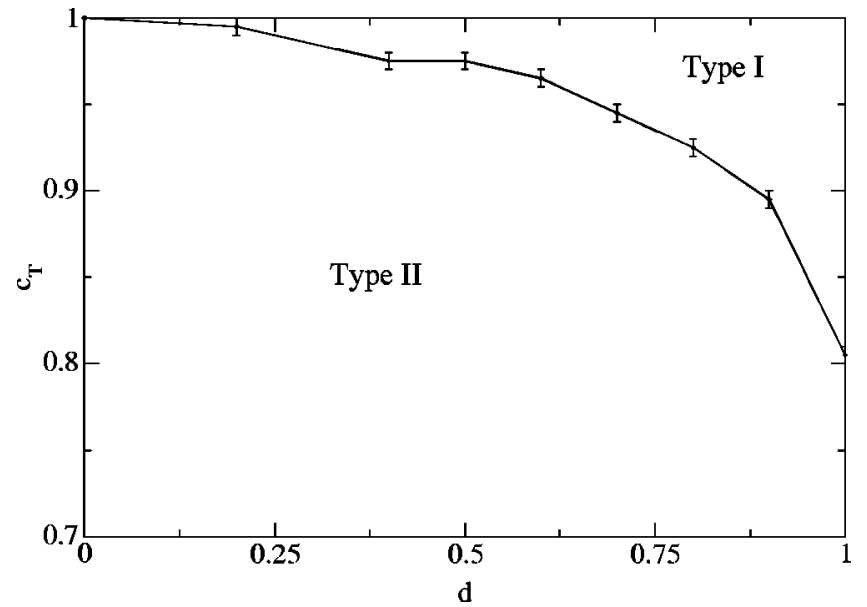

FIG. 7. $c_{T}$ vs $d$, for an initial condition of the form Eq. (12) and surface tension $B=1 / 14$.

$$
\frac{\dot{a}_{2}(t)}{a_{2}(t)}=\frac{6}{7}\left\{1+4 a_{2}^{2}(t)\right\} .
$$

From these equations it can be observed that the viscosity contrast reinforces the growth of the mode $k=1$ through a quadratic coupling with the mode $k=2$. On the contrary, for $c$ close to zero this quadratic term is small and the cubic term is negative, thus weakening the growth of the mode $k=1$ in front of the mode $k=2$. Hence, in the weakly nonlinear regime the reinforced growth of $a_{1}(t)$ pushes the interface towards the single finger configuration for $c$ large, and for $c$ small the growth of $a_{1}(t)$ is weakened and the dynamics tends to the two-finger configuration.

A change in the surface tension value yields qualitatively similar results, but now the two initial modes have different growth rates and the interface can suffer significant changes even in the linear regime: A second bump can develop from a configuration which initially had one bump if $B<1 / 7$, or a bump of a two-bump configuration can be suppressed if $B$ $>1 / 7$. Then, from this simple linear regime considerations one can infer that a plot of $c_{T}$ versus $d$ will have a major difference from the plot depicted in Fig. 6: For $d=1$ (single bump) $c_{T}$ will be greater than zero if $B<1 / 7$. In Fig. 7, $c_{T}$ versus $d$ is plotted for $B=1 / 14$. As predicted, $c_{T}(d=1)$ is larger than zero and for this value of $B$ it is closer to 1 than to 0 . However, one must keep in mind that for $B \neq 1 / 7$ the linear growth rates for the two modes are different, and consequently the initial condition Eq. (12) is not described by a single parameter in the linear regime. Then, if we had computed $c_{T}(d)$ with different values of the ratio $a_{1} / a_{2}$ we would have obtained a curve different from the one of Fig. 7, but qualitatively equivalent. On the other hand, if $B>1 / 7$ $c_{T}(d)$ will reach zero for $d<1$.

Figures 6 and 7 show that type II dynamics occupies the larger part of the phase diagrams. In particular, for low values of $d$ the behavior of the system is type II except for viscosity contrasts very close to one. Taking into account that the fingers arising spontaneously from the linear instability of the planar interface have similar length, that is, $d$ is close to zero in real experiments, type II dynamics seems to be the dominant behavior as long as the viscosity contrast is slightly or well below one. Thus, in situations with arbitrary viscosity contrast, finger competition is generically absent or weak, and the ST finger may not be reached. Instead, a more complex situation arises, and attractors absent for high viscosity contrast appear. This will be discussed in the section below.

\section{B. Taylor-Saffman bubbles: The competing attractors}

We have observed that within type II behavior, in certain cases the leading finger evolves for long times to a configuration consisting of a bubble-shaped tip connected to the rest of the interface by a long, narrow neck, that can be extremely thin next to the bubble region. This bubble formation process has been observed for a wide range of values of the viscosity contrast, except for values very close to 1 . Formation of bubbles for low viscosity contrast has been previously reported by Ref. 51 in more complex interfacial configurations. Bubble shaped (closed) exact solutions are known for $B$ $=0,{ }^{75}$ and similarly to the ST finger, bubbles are also solutions with finite $B$ via a similar selection mechanism.

A detailed study of the long time asymptotics of type II dynamics and, in particular, the issue of whether the dynamics leads to finite-time pinch-off, is much beyond the scope of this section, and is indeed one of the future directions to be explored. The basic idea we would like to push here is that, regardless of whether or not there is finite-time pinchoff, it seems clear that the isolated bubble solutions do behave in practice as attractors of the dynamics, at least partially. The fact that the attractor of the dynamics may have a different topology than the initial interface is an interesting and unusual situation which in a sense explains, in the language of dynamical systems, why the tendency to pinch-off is observed so often and indeed generically in the problem.

Taylor and Saffman ${ }^{75}$ found a two-parametric family of exact solutions of the problem with zero-surface tension consisting of symmetric bubbles advancing with constant velocity $\mathcal{U}$. Its functional form is

$$
\begin{aligned}
x= & \frac{2}{\pi} \frac{\mathcal{U}-1}{\mathcal{U}} \tanh ^{-1}\left[\sin ^{2}\left(\frac{\pi}{2} \mathcal{U} \lambda\right)\right. \\
& \left.-\cos ^{2}\left(\frac{\pi}{2} \mathcal{U} \lambda\right) \tan ^{2}\left(\frac{\mathcal{U}}{4} y-\frac{\pi}{2} \mathcal{U}\right)\right]^{1 / 2},
\end{aligned}
$$

and contains two parameters, the (dimensionless) bubble velocity ${ }^{88} \mathcal{U}$ and the maximum width $\lambda$ of the bubble (measured in channel-width units). The area $S$ bounded by the interface reads ${ }^{75}$

$$
S=16 \frac{\mathcal{U}-1}{\mathcal{U}^{2}} \tanh ^{-1}\left[\tan ^{2}\left(\frac{\pi}{4} \mathcal{U} \Lambda\right)\right] .
$$

In the limit $\mathcal{U} \lambda \rightarrow 1$ with $\mathcal{U}$ fixed, the area $S$ of the bubble diverges and the steady-state Saffman-Taylor finger solution is recovered. The area of the bubble does not specify $\mathcal{U}$ and $\lambda$ since Eq. (17) only provides one relation between them, and there exists a continuum of solutions with arbitrary speed that satisfy the area condition. Thus, we encounter a selection problem fully analogous to the classical finger-width selection problem, where the zero-surface tension solution for a steadily translating Saffman-Taylor finger has an arbi- 


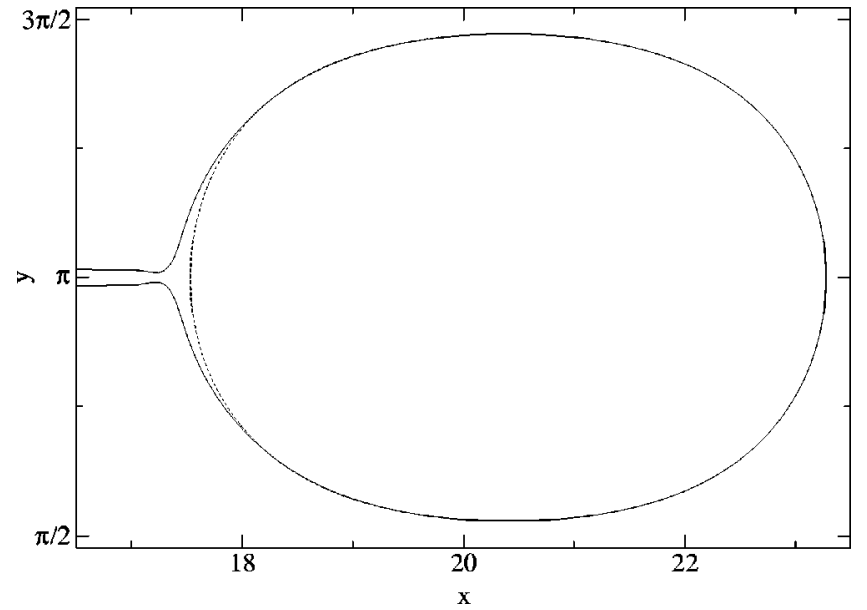

FIG. 8. Bubble region of the computed interface with initial condition $a_{1}$ $=0.038223, a_{2}=0.00972855, c=0.5$ and $B=0.01$ together with the interface obtained from Eq. (18) with $\mathcal{U}=1.975$ and $\alpha=0.8969$.

trary width. Tanveer ${ }^{76,77}$ showed that the introduction of a finite surface tension removes the degeneracy in the bubble speed $\mathcal{U}$, and that families of bubbles that do not contain the symmetries present in the solution Eq. (16) exist.

Since the bubble-shaped region of the interface that forms for some parameter values resembles the TaylorSaffman bubble solution, we have compared the bubble region of the computed interface with finite $B$ and the bubble given by solution Eq. (16). For convenience the conformal mapping version of Eq. (16) is used. The bubble shape in terms of the complex variable $z=x+i y$ reads $^{77}$

$$
z(s)=\ln \left(\frac{e^{i s}-\alpha}{e^{i s}+\alpha}\right)+\left(\frac{2}{\mathcal{U}}-1\right) \ln \left(\frac{1+e^{i s} \alpha}{1-e^{i s} \alpha}\right)+i \pi,
$$

where the constant parameter $\alpha$ takes values in the range $(0,1)$ and the interface shape is described by $0<s<2 \pi$. The interface width is $2 \pi \lambda$ and the bubble is centered along the mid-channel axis. The parameter $\alpha$ relates to $\lambda$ and $\mathcal{U}$ through the equation

$$
\lambda=\frac{1}{\pi} \frac{4}{\mathcal{U}} \tan ^{-1}\left(\frac{2 \alpha}{1-\alpha^{2}}\right) .
$$

In Fig. 8 the bubble region of an evolution with $c=0.5$ and $B=0.01$ is plotted together with the analytical bubble with $\mathcal{U}=1.975$ and $\alpha=0.8969$. The agreement between the two curves is extremely accurate, except for the neighborhood of the neck connection. For larger values of surface tension, the agreement is still remarkably good. The excellent agreement between the bubble region of the computed interface and the Taylor-Saffman bubble is a strong indication that the interface is being (locally) attracted to the Taylor-Saffman bubble fixed point. In addition, this also suggests that the dynamics of the bubble-shaped region is almost independent of the rest of the interface. Note, however, that through the neck that connects the two parts of the interface there is a residual finite flux of fluid that allows a slight increase of the bubble area. This variation is slow enough to keep it very close to a stationary solution on the time scale of interface displacement.

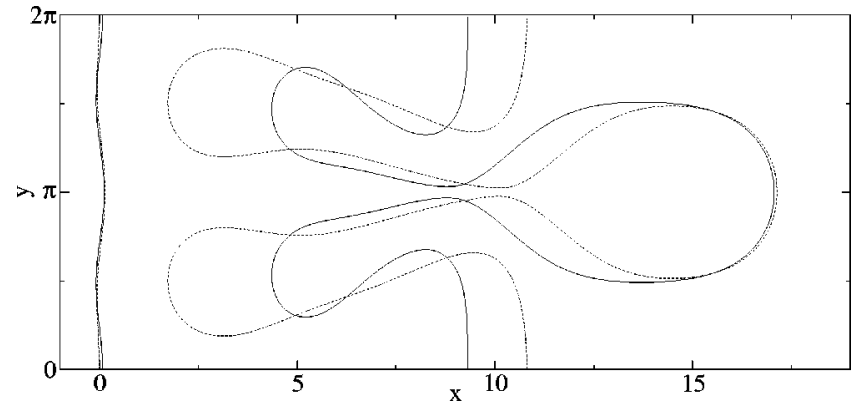

FIG. 9. Evolution of two different initial conditions with $a_{1}=0.02, a_{2}$ $=0.08972$ (solid line) and $a_{1}=0.07, a_{2}=0.05988$ (dashed line). The viscosity contrast is $c=0.8$ and surface tension is $B=1 / 14$. The final times are $t=9.25$ (solid line) and $t=8.0$ (dashed line) in some dimensionless units.

The area of the bubble shaped region, for a given $B$ and $c$ depends also on the particular initial condition. This is illustrated in Fig. 9, where the evolution of two different initial conditions but with same values of $B$ and $c$ is plotted. The area of the two bubble shaped areas, although not completely formed yet, is clearly distinct, showing that the two evolutions are being attracted, at least during a certain time, to different bubble fixed points. Apparently, the area of the bubble changes continuously with the initial condition, and the bubble region is being attracted to different points of the continuum of Taylor-Saffman solutions. As a general rule, our computations show that, for a given initial condition, as the viscosity contrast is decreased, the area of the bubble gets smaller. A possible explanation for this behavior and more details of this discussion can be found in Refs. 46 and 74.

While many interesting questions remain open, most remarkably those concerning finite-time pinch-off, the conclusions of this analysis are clear. On the one hand, the dynamics of low viscosity contrast seems to be more generic than that (most usual in the literature) of high viscosity contrast, in the sense that only for values of $c$ very close to one the standard finger competition scenario is typical. There is thus a very strong sensitivity of the dynamics to $c$ in the neighborhood of $c=1$. On the other hand, we have reinforced the conjecture that there is a continuous reduction of the basin of attraction of the Saffman-Taylor with $c$. Furthermore, we have identified the isolated Taylor-Saffman bubbles as the missing attractors which compete with the Saffman-Taylor finger. Having a different topology than that of the interface in the initial configuration, the generic tendency to pinch-off observed in experiments and simulation finds a natural explanation.

\section{Rotating Hele-Shaw flows}

A variation of the traditional viscous fingering problem in radial geometry which has been studied more recently is that of rotating Hele-Shaw flows. This has been explored theoretically following parallel steps to the case of channel geometry. It was shown that exact solutions for the case of zero surface tension can be found and that rotation, under certain circumstances, may prevent the formation of cusp singularities. ${ }^{62}$ The weakly nonlinear analysis has also been extended to this case. ${ }^{59}$ On the experimental side a series of 


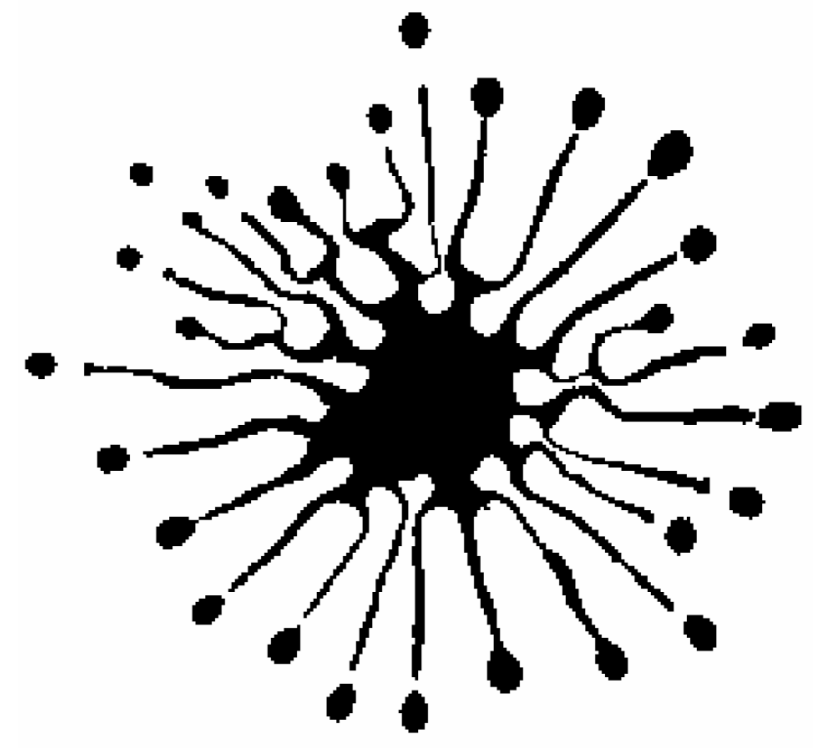

FIG. 10. Typical experimental pattern of low viscosity contrast fingering under rotation. Note that fingers do not appear to compete and develop narrow filaments which lead to experimental pinch-off.

works of Ortín and co-workers have explored systematically several aspects of rotating Hele-Shaw flows. ${ }^{78-82}$

One of the interests of this experimental setup is the possibility to explore the region of low viscosity contrast while driving the instability by density contrast, in analogy with gravity-driven viscous fingering in channel geometry. ${ }^{81}$ The lack of competition in this case leads to completely different morphologies than those observed for high viscosity contrast (see an example in Fig. 10). Both cases, however differ even more strongly from the traditional fingering patterns for fluid injection. An exhaustive experimental study of low viscosity contrast fingering in a rotating Hele-Shaw cell can be found in Ref. 81 .

The importance of wetting effects on the boundary conditions at the interface has also been made clear in the case of rotating Hele-Shaw flows, for instance giving rise to very different patterns depending on whether the cell is prewetted or not ${ }^{82,83}$ (see an example in Fig. 11). The traditional boundary condition has been shown to be valid in general if the cell has been uniformly wetted by a thin film of the wetting fluid. Otherwise, if the wetting fluid displaces the nonwetting one in a dry cell, the resulting patterns differ significantly. The need to incorporate the physics of the motion of contact lines in the boundary condition then brings up a new microscopic length scale into the problem (related to the thickness of the precursor wetting layer) which, remarkably enough, fixes some basic morphological features of the macroscopic pattern. This point is now a promising direction of current research.

Another important aspect that has originated in the study of rotating Hele-Shaw flows is that of topological singularities. While the tendency to pinch-off has already been demonstrated in low-contrast fingering in the channel geometry, such phenomenon is strongly enhanced by rotation (see Fig. 10). This has been worked out in detail in terms of viscosity

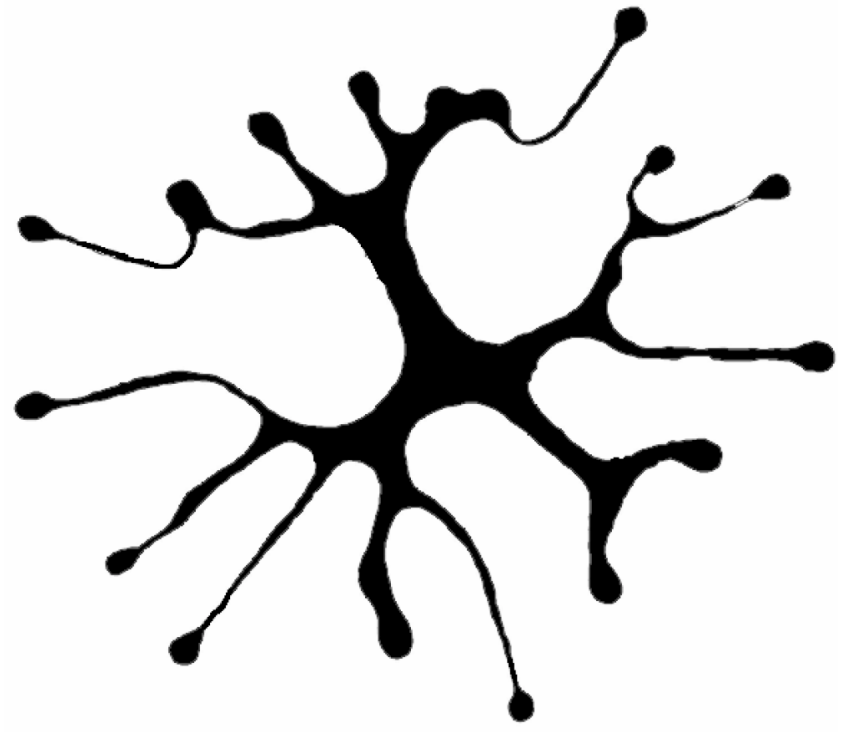

FIG. 11. Typical experimental pattern for $c=1$, with air as the outer fluid, if the cell is initially dry.

contrast. While in rotating Hele-Shaw flows it can be proven that interface pinch-off occurs at least at infinite time, the detailed study of the pinch-off itself, in particular for the case of two viscous fluids, is inherently different from that of ordinary Hele-Shaw flows and is one of the interesting problems that is currently under study. ${ }^{82,84}$

\section{SUMMARY AND PERSPECTIVES}

In this article we have briefly reviewed some of the recent results on the study of viscous fingering after the full understanding of the steady state selection problem. Since then, the interest has focussed primarily on the dynamics of fingering patterns. A leading idea of this article has been the fact that the dynamics of this highly nonlinear and nonlocal free-boundary problem hides surprising and nontrivial dynamical features which challenge our intuition and question our qualitative understanding of Laplacian screening as the basic mechanism of finger competition and growth in this class of problems.

At the level of the ST instability itself we have recalled very recent systematic extensions of the linear stability analysis to the weakly nonlinear regime, and the identification of a class of exact solutions with finite surface tension, the elastica solutions, as the unstable subcritical branch of the ST instability. In the experimental test of the resulting nonlinear ST instability, a new experimental procedure has been developed to generate arbitrary interface configurations for the initial condition. This opens a wide range of possibilities to explore dynamical behaviors in a direct way that was not conceivable before.

Concerning the role of surface tension we have reviewed the basic leitmotif of a series of contributions aiming at elucidating the possible extension to the dynamics of the traditional solvability theory. The remarkable obtention of broad classes of exact time-dependent solutions for zero surface tension was the starting point. The use of concepts of dy- 
namical systems theory, together with the extension of static solvability theory to multifinger configurations, leaded us to the define dynamic solvability as the scenario in which surface tension unfolds the structurally unstable phase flow associated to the degeneracy of multifinger stationary solutions, restoring hyperbolicity of multifinger fixed points and, consequently modifying essentially the topology of the phase space flow in regions far apart from the attractor itself. Formally, this phenomenon makes contact with static (traditional) solvability theory in the analysis that isolates a discrete set out of a continuum of stationary solutions (also a process of restoring hyperbolicity in the single-finger selection case). However, we call it dynamic because of the qualitative modification of the global phase space flow (phase portrait) as a consequence of the saddle-point structure unfolded for the surviving multifinger solution.

The pursuit of this idea and the completion of the general picture arising from the comparison of the dynamics with strictly zero and with vanishingly small surface tension, has been possible thanks to the asymptotic theory developed by Tanveer and co-workers. Their main conclusion, namely that after a time of order one the two dynamics are significantly different, has been put to the test and has shown spectacular divergences between trajectories evolving from the same initial conditions but with the two dynamics above (with and without surface tension) in sufficiently generic regions of phase space.

The general picture for the comparison of the dynamics with and without surface tension is thus as follows. In general, the dynamics with zero surface tension can only be a good approximation of that with a small surface tension (regular perturbation) for a time of order unity, and it is in general completely wrong after that time (which can be determined in terms of the dynamics of zero surface tension). The dramatic effect on the structure of the phase space flow, which we call dynamic solvability, is only manifest after that time of order unity. Most remarkably, even families of trajectories evolving towards the same attractor (the singlefinger solution predicted by static solvability) with both dynamics may follow phase space paths completely apart from each other. This occurs for finite-measure sets of initial conditions and clearly points out to an inherently dynamic, singular effect of surface tension. We thus see that the global restructuring of phase space introduced by surface tension goes much beyond the modification of local flow structure around the fixed points (as for static solvability), and therefore, the qualification of dynamic solvability makes full sense.

Another class of results which question the naive picture of finger competition are those referring to the dynamical role of viscosity contrast. We have shown that the basin of attraction of the Saffman-Taylor finger is only the full phase space for the strict limit of high viscosity contrast $(c=1)$, while it decreases gradually with $c$ to a small but not vanishing region for $c=0$. The maximum sensitivity to $c$ is precisely at $c=1$, while the behavior for lower $c$, for which no finger competition is observed, must be considered as more generic. The (partial) attractors of the dynamics which compete with the ST finger have been identified as closed bubble solutions. The fact that these have a different topology than the initial condition provides an explanation of the observed tendency to interface pinch-off.

The effect of viscosity contrast has only been studied for dimensionless surface tension of order one. It remains an open and challenging question to explore how the perturbative picture of small surface tension is modified if viscosity contrast is different from $c=1$. While classical (static) solvability theory is not fundamentally modified by varying $c$, the situation for the time-dependent behavior is expected to be much more involved, as suggested by the lack of explicit time-dependent solutions for $B=0$ and $c \neq 1$. To our knowledge the only exact time-dependent solution for arbitrary $c$ $(B=0)$ is the (time-dependent) single-finger ST finger of relative width $\lambda=1 / 2 .{ }^{85}$ Remarkably, other filling fractions $\lambda$ have time-dependent single-finger solutions only for $c=1 .^{46,58}$ This result unveils an intriguing connection between the width selection problem and the dynamical role of viscosity contrast.

The case of rotating Hele-Shaw flows has been studied only quite recently both theoretically and experimentally and has revealed a wealth of new phenomena and new interesting questions. Apart from the interplay between dynamics and interface morphology, the most salient feature in terms of future research has been the enhanced occurrence of topological singularities and their relation to viscosity contrast. While pinch-off singularities have been studied in HeleShaw problems in the past, it was usually in rather particular setups, specifically designed to produce pinch-off. In the case of rotating Hele-Shaw flows, however, it has been shown that the dynamics leads naturally to situations approaching pinchoff. However, a detailed study of the asymptotic approach to pinch-off within a lubrication approximation is still lacking. This point is currently being explored both analytically and numerically. ${ }^{84}$

The study of rotating flows has also pointed out the need for a more careful study of the effective boundary condition at the interface when the wetting fluid is displacing the nonwetting one, a situation that is usual for centrifugally driven flows but that is atypical in more traditional experiments. In the former case, having the cell prewetted makes a real difference. The description of the wetting fluid advancing in a dry cell relates then to the motion of a contact line, a common but generally unsolved problem in fluid mechanics. A very exciting preliminary study shows that, even with a rather crude description of the contact line motion, the effect is not only appreciable quantitatively but also qualitatively in the overall morphology of the resulting patterns. The treatment of the contact line requires indeed the introduction of a microscopic length scale (related to the thickness of the precursor wetting film). It is thus remarkable, in a way that is reminiscent of the effect of the capillary length on pattern selection in the microscopic solvability scenario, that such a microscopic length scale has a drastic effect on the macroscopic pattern morphology. This open question is also of great interest and also sets forth a promising future perspective.

Finally, one of the most interesting lines of future research in the context of Hele-Shaw flows consists in adding 
controlled modifications of the classical setup to define richer model systems, and use them to pursue basic and general principles of more complicated systems, in the spirit of Ref. 49. The case of the random Hele-Shaw cell as a model of a porous medium is a paradigmatic and fruitful recent example. ${ }^{46,50}$ This particular case has leaded to an $a b$ initio derivation of an interface equation, which has allowed to draw general conclusions on the problem of interface roughening in fluid invasion of porous media, and also to give an appropriate theoretical framework where renormalization group analysis and numerical simulation can work on firm grounds. The usefulness of the Hele-Shaw-type modelsystem analysis has thus been remarkable in a field where controversy and confusion has been too common. Not only the derivation of the exact interface equation with all noise contributions allows reinterpretation of old existing experimental and simulational results, but most importantly, the explicit knowledge of all parameters of the interface equation allows one to design new experiments with the optimal choice of parameters. Experiments on Hele-Shaw cells with random gap as a model system for porous media have already been carried out by Ortín and co-workers (see, for instance, Ref. 86). The possibility of a clear connection between theory and experiment and the good control which is possible in both aspects makes this line also a promising and exciting direction.

In summary, after several decades, viscous fingering and its variations continue to be a challenging and eversurprising model system. Not only is it instrumental for exploring fundamental physics, but it yields a fertile framework where analytical insight and controlled experiments can shed light to other problems in interfacial pattern formation.

\section{ACKNOWLEDGMENTS}

I am grateful to D. Jasnow and J. V. Maher for introducing me to the subject of viscous fingering. A large part of the work and ideas here presented have been developed in collaboration with my Ph.D. students F. X. Magdaleno, R. Folch, E. Pauné, and E. Alvarez-Lacalle. I also thank J. Ortín for close collaboration in the experimental aspects of this work. Financial support from the MCyT (Spain), Project No. BQU2003-05042-C02-02 and the European Commission, Project Ref. HPRN-CT-2002-00312 is acknowledged.

${ }^{1}$ H. Hele-Shaw, Nature (London) 58, 33 (1898).

${ }^{2}$ P. Saffman and G. Taylor, Proc. R. Soc. London, Ser. A 245, 312 (1958).

${ }^{3}$ G. Homsy, Annu. Rev. Fluid Mech. 19, 271 (1987).

${ }^{4}$ D. Bensimon, L. Kadanoff, S. Liang, B. I. Shraiman, and C. Tang, Rev. Mod. Phys. 58, 977 (1986).

${ }^{5}$ Y. Couder, in Perspectives in Fluid Dynamics, edited by H. M. G. K. Batchelor and M. Worster (Cambridge University Press, Cambridge, 2000).

${ }^{6}$ S. Tanveer, J. Fluid Mech. 409, 273 (2000).

${ }^{7}$ J. Casademunt and F. X. Magdaleno, Phys. Rep. 337, 1 (2000).

${ }^{8}$ W. F. Engelberts and L. J. Klickenberg, Petr. Congr. Third World, p. 544 (1951).

${ }^{9}$ J. S. Langer, in Chance and Matter, 1986 Les Houches Lectures, edited by J. Souletie, J. Vannimenus, and R. Stora (North-Holland, Amsterdam, 1987).

${ }^{10}$ D. A. Kessler, J. Koplik, and H. Levine, Adv. Phys. 35, 255 (1988)

${ }^{11}$ Dynamics of Curved Fronts, Perspectives in Physics, edited by P. Pelcé (Academic, Boston, 1988).
${ }^{12}$ E. A. Brener and V. I. Mel'nikov, Adv. Phys. 40, 53 (1991).

${ }^{13}$ Y. Pomeau and M. B. Amar, in Solids Far from Equilibrium, edited by C. Godrèche (Cambridge University Press, Cambridge, 1992).

${ }^{14}$ J. Gollub and J. Langer, Rev. Mod. Phys. 71, S396 (1999).

${ }^{15}$ S. Howison, J. Fluid Mech. 167, 439 (1986).

${ }^{16}$ S. Howison, SIAM (Soc. Ind. Appl. Math.) J. Appl. Math. 46, 20 (1986).

${ }^{17}$ S. Howison, Eur. J. Appl. Math. 3, 209 (1992).

${ }^{18}$ S. Tanveer, Philos. Trans. R. Soc. London, Ser. A 343, 155 (1993).

${ }^{19}$ V. Entov, P. Etingof, and D. Klinbock, Eur. J. Appl. Math. 6, 399 (1995).

${ }^{20}$ S. Howison, J. Fluid Mech. 409, 243 (2000).

${ }^{21}$ E. Elezgaray, Phys. Rev. E 57, 6884 (1998).

${ }^{22}$ E. Elezgaray, Philos. Mag. B 78, 159 (1998).

${ }^{23}$ R. L. Chuoke, P. van Meurs, and C. van der Poel, Pet. Trans. AIME 216, 188 (1959).

${ }^{24}$ W. Mullins and R. Sekerka, J. Appl. Phys. 35, 444 (1964).

${ }^{25}$ G. Ivantsov, Dokl. Akad. Nauk SSSR 58, No. 4 (1947).

${ }^{26}$ D. C. Hong and J. S. Langer, Phys. Rev. Lett. 56, 2032 (1986).

${ }^{27}$ B. I. Shraiman, Phys. Rev. Lett. 56, 2028 (1986)

${ }^{28}$ R. Combescot, T. Dombre, V. Hakim, Y. Pomeau, and A. Pumir, Phys. Rev. Lett. 56, 2036 (1986).

${ }^{29}$ S. Tanveer, Phys. Fluids 30, 2318 (1987).

${ }^{30}$ M. B. Hastings, Phys. Rev. E 55, 135 (1997).

${ }^{31}$ M. B. Hastings and L. S. Levitov, Physica D 116, 244 (1998),

${ }^{32}$ E. Somfai, L. Sander, and R. Ball, Phys. Rev. Lett. 83, 5523 (1999).

${ }^{33}$ B. Davidovitch, H. G. E. Hentshel, Z. Olami, I. Procaccia, L. M. Sander, and E. Somfai, Phys. Rev. E 59, 1368 (1999).

${ }^{34}$ B. Davidovitch, M. Feigenbaum, H. Hentshel, and I. Procaccia, cond-mat/ 0002420 (unpublished).

${ }^{35}$ B. Davidovitch and I. Procaccia, cond-mat/0003044 (unpublished).

${ }^{36}$ F. Barra, B. Davidovitch, A. Levermann, and I. Procaccia, Phys. Rev. Lett. 87, 134501 (2001).

${ }^{37}$ M. Mineev-Weinstein, P. Wiegmann, and A. Zabrodin, Phys. Rev. Lett. 84, 5106 (2000)

${ }^{38}$ O. Agam, E. Bettelheim, P. Wiegman, and A. Zabrodin, Phys. Rev. Lett. 88, 236801 (2002).

${ }^{39}$ E. Lajeunesse and Y. Couder, J. Fluid Mech. 419, 125 (2000).

${ }^{40}$ A. Pereira, Ph.D. thesis, Université de Bordeaux I (2004).

${ }^{41}$ M. Siegel, S. Tanveer, and W.-S. Dai, J. Fluid Mech. 323, 201 (1996).

${ }^{42}$ M. Siegel and S. Tanveer, Phys. Rev. Lett. 76, 419 (1996).

${ }^{43}$ F. X. Magdaleno and J. Casademunt, Phys. Rev. E 57, R3707 (1998).

${ }^{44}$ E. Pauné, F. X. Magdaleno, and J. Casademunt, Phys. Rev. E 65, 056213 (2002).

${ }^{45}$ E. Pauné, M. Siegel, and J. Casademunt, Phys. Rev. E 66, 046205 (2002).

${ }^{46}$ E. Pauné, Ph.D. thesis, Universitat de Barcelona (2002).

${ }^{47}$ J. Casademunt and D. Jasnow, Phys. Rev. Lett. 67, 3677 (1991).

${ }^{48}$ J. Casademunt and D. Jasnow, Physica D 79, 387 (1994).

${ }^{49}$ K. V. McCloud and J. V. Maher, Phys. Rep. 260, 139 (1995).

${ }^{50}$ E. Pauné and J. Casademunt, Phys. Rev. Lett. 90, 144504 (2003).

${ }^{51}$ G. Tryggvason and H. Aref, J. Fluid Mech. 136, 1 (1983).

${ }^{52}$ P. G. Saffman, Q. J. Mech. Appl. Math. 12, 146 (1959).

${ }^{53}$ S. Ponce Dawson and M. Mineev-Weinstein, Physica D 73, 373 (1994).

${ }^{54}$ T. Hou, J. Lowengrub, and M. Shelley, J. Comput. Phys. 114, 312 (1994).

${ }^{55} \mathrm{H}$. Ceniceros, T. Hou, and H. Si, Phys. Fluids 11, 2471 (1999).

${ }^{56}$ H. Ceniceros and T. Hou, J. Fluid Mech. 409, 251 (2000).

${ }^{57}$ Asymptotics Beyond All Orders, Nato ASI Series, Series B: Physics, edited by H. Segur, S. Tanveer, and H. Levine (Plenum, New York, 1991).

${ }^{58}$ E. Alvarez-Lacalle, J. Casademunt, and J. Ortín, Phys. Rev. E 64, 016302 (2001).

${ }^{59}$ E. Alvarez-Lacalle, E. Pauné, J. Casademunt, and J. Ortín, Phys. Rev. E 68, 026308 (2003).

${ }^{60}$ E. Alvarez-Lacalle, J. Ortín, and J. Casademunt, Phys. Rev. Lett. 92, 054501 (2004)

${ }^{61}$ J. Nye, H. Lean, and A. Wright, Eur. J. Phys. 5, 73 (1984).

${ }^{62}$ F. X. Magdaleno, A. Rocco, and J. Casademunt, Phys. Rev. E 62, R5887 (2000).

${ }^{63}$ J. Casademunt and F. X. Magdaleno, Phys. Rev. Lett. 81, 5950 (1998).

${ }^{64}$ F. X. Magdaleno and J. Casademunt, Phys. Rev. E 60, R5013 (1999).

${ }^{65}$ J. Guckenheiner and P. Holmes, Nonlinear Oscillations, Dynamical Systems and Bifurcations of Vector Fields, Applied Mathematical Sciences (Springer-Verlag, New York, 1983).

${ }^{66}$ D. A. Kessler and H. Levine, Phys. Rev. A 33, 3625 (1986).

${ }^{67}$ S. Tanveer, G. Baker, and M. Fisher, J. Comput. Phys. 120, 348 (1994).

${ }^{68}$ D. A. Kessler and H. Levine, Phys. Rev. Lett. 86, 4532 (2001). 
${ }^{69}$ J. V. Maher, Phys. Rev. Lett. 54, 1498 (1985).

${ }^{70}$ G. Tryggvason and H. Aref, J. Fluid Mech. 154, 287 (1985).

${ }^{71}$ S. A. Curtis and J. V. Maher, Phys. Rev. Lett. 63, 2729 (1989).

${ }^{72}$ J. Viñals and D. Jasnow, Phys. Rev. A 46, 7777 (1992).

${ }^{73}$ J. Eggers, Rev. Mod. Phys. 69, 865 (1997).

${ }^{74}$ E. Pauné and J. Casademunt (in preparation).

${ }^{75}$ G. Taylor and P. Saffman, Q. J. Mech. Appl. Math. 12, 265 (1959).

${ }^{76}$ S. Tanveer, Phys. Fluids 29, 3537 (1986).

${ }^{77}$ S. Tanveer, Phys. Fluids 30, 651 (1987).

${ }^{78}$ L. Carrillo, F. X. Magdaleno, J. Casademunt, and J. Ortín, Phys. Rev. E 54, 6260 (1996).

${ }^{79}$ L. Carrillo, J. Soriano, and J. Ortín, Phys. Fluids 11, 778 (1999).

${ }^{80}$ L. Carrillo, J. Soriano, and J. Ortín, Phys. Fluids 12, 1685 (2000).
${ }^{81}$ E. Alvarez-Lacalle, J. Ortín, and J. Casademunt, Phys. Fluids 16, 908 (2004).

${ }^{82}$ E. Alvarez-Lacalle, Ph.D. thesis, Universitat de Barcelona (2004).

${ }^{83}$ E. Alvarez-Lacalle, J. Ortín, and J. Casademunt (in preparation).

${ }^{84}$ R. Folch, E. Alvarez-Lacalle, J. Ortín, and J. Casademunt, physics/ 0408096 (unpublished).

${ }^{85}$ P. Jacquard and P. Séguier, J. Mec. 1, 367 (1962).

${ }^{86}$ J. Soriano, J. Ortín, and A. Hernández-Machado, Phys. Rev. E 66, 031603 (2002).

${ }^{87} \mathrm{~A}$ rescaling of the interface height by a factor $F$ is compensated by shifting the initial time an amount $\frac{7}{6} \ln F$.

${ }^{88}$ If $c \neq 1$ the bubble velocity $\mathcal{U}$ has to be replaced in Eq. (16) by a more complicated expression, that can be found in Ref. 75 . 